\title{
Lymphocytes Stimulate Expression of 5-Lipoxygenase and Its Activating Protein in Monocytes In Vitro Via Granulocyte Macrophage Colony-stimulating Factor and Interleukin 3
}

\author{
William L. Ring, Carl A. Riddick, Joseph R. Baker, Dominic A. Munafo, and Timothy D. Bigby \\ Division of Pulmonary and Critical Care Medicine, Department of Medicine, University of California, San Diego, and Department of \\ Veterans Affairs Medical Center, San Diego, California 92161
}

\begin{abstract}
The aim of this study was to examine the role of lymphocytes in regulating expression of the 5-lipoxygenase pathway in monocytes. When monocytes were cultured over a period of days with lymphocytes, calcium ionophore-stimulated 5-lipoxygenase activity was enhanced. If lymphocytes alone were activated with lectins and their supernatants added to monocytes, stimulated 5-lipoxygenase activity was increased, whereas supernatants from lymphocytes cultured without lectins had no effect. Increased immunoreactive protein and mRNA for 5-lipoxygenase and 5-lipoxygenase activating protein were present in cells conditioned with lectin-activated lymphocyte supernatants. The effect of activated-lymphocyte supernatants could be mimicked by either GM-CSF or IL-3, but there was no additive effect with both cytokines. Both GM-CSF and IL-3 were present in the supernatant from lectin-activated lymphocytes at concentrations above their $\mathrm{ED}_{50}$, but were undetectable in the supernatant from nonactivated lymphocytes. The effect of lectin-activated lymphocyte supernatant could be inhibited by neutralizing antibodies to both cytokines, but not to either cytokine alone. We conclude that lymphocytes can regulate the expression of 5-lipoxygenase in monocytes, over a period of days, via the release of soluble factors, primarily GM-CSF and IL-3. (J. Clin. Invest. 1996. 97:1293-1301.) Key words: arachidonic acid • leukotriene - inflammation • cytokines $\bullet$ macrophages
\end{abstract}

\section{Introduction}

Leukotrienes are oxygenated metabolites of arachidonic acid derived from the 5-lipoxygenase pathway and are potent inflammatory mediators implicated in a number of diseases (1). After the release of arachidonic acid from membrane phospholipids, the enzyme 5-lipoxygenase is responsible for catalyzing the first two steps in this pathway. Initially, it converts arachidonic acid to 5(S)-hydroperoxy-6,8-trans-11,14-cis-eicosatetraenoic acid (5-HPETE) which is then either degraded to the metabolite 5(S)-hydroxy-6-8,11,14,-cis-eicosatetraenoic acid $(5 \text {-HETE })^{1}$ or is further acted upon by 5 -lipoxygenase to form

Address correspondence to Timothy D. Bigby, M.D., University of California, San Diego, 111-J, Department of Veterans Affairs Medical Center, 3350 La Jolla Village Dr., San Diego, CA 92161. Phone: 619-552-8585x3541; FAX: 619-546-1754; E-mail: tbigby@UCSD.edu

Received for publication 9 October 1995 and accepted in revised form 14 December 1995.

The Journal of Clinical Investigation

Volume 97, Number 5, March 1996, 1293-1301 the unstable epoxide intermediate 5(S)-5,6-oxido-7,9-trans11,14-cis-eicosatetraenoic acid (leukotriene $\mathrm{A}_{4}$; $\mathrm{LTA}_{4}$ ). Within the cell, the synthesis of 5-lipoxygenase products is dependent on the nuclear membrane-associated protein, 5-lipoxygenaseactivating protein (FLAP) (2-4).

Specific control of 5-lipoxygenase and FLAP expression has long been suspected because expression of 5-lipoxygenase activity is tissue specific and narrowly distributed to inflammatory cells (5). Moreover, there is wide variation in 5-lipoxygenase activity and in 5-lipoxygenase and FLAP levels even among different inflammatory cell types. For example, lung macrophages have 10-fold greater 5-lipoxygenase activity than peripheral blood monocytes on a per cell basis (6), and this increased activity is associated with greater quantities of 5-lipoxygenase and FLAP per cell $(2,7)$. However, we have reported recently that peripheral blood monocytes lose 5-lipoxygenase activity when cultured over days in $10 \%$ autologous serum, in spite of morphological changes consistent with differentiation into macrophages (7a). This loss in activity is associated with decreases in protein and mRNA for both 5-lipoxygenase and FLAP (7a). These findings suggest that increased capacity for 5-lipoxygenase product synthesis is not an intrinsic component of the maturation process of monocytes to macrophages. Rather, they suggest that extrinsic factor(s) are present in vivo that are absent from our in vitro model.

Several studies have examined aspects of 5-lipoxygenase pathway regulation. A myeloid precursor cell line, HL-60, can be differentiated into granulocytic, monocytic, or macrophagelike cells by a number of agents, including DMSO, retinoic acid, dibutyryl cAMP, and 1,25-dihydroxyvitamin $\mathrm{D}_{3}$. This differentiation is associated with changes in the expression of both 5-lipoxygenase and FLAP (8). These changes in HL-60 cells are, at least in part, caused by TGF- $\beta$ in serum (9). In the mononuclear cell line, U937, and in HL-60 cells, oxidized low density lipoprotein increases 5-lipoxygenase activity by increasing expression of FLAP (10). 5-Lipoxygenase activity in rat lung macrophages can be transiently enhanced twofold by IFN- $\gamma$ in vitro, but this effect is lost by $24 \mathrm{~h}$ (11).

Additional studies have been performed on primary isolates of human inflammatory cells. Human eosinophils cultured for $7 \mathrm{~d}$ in the presence of the mouse $3 \mathrm{~T} 3$ fibroblast cell line and either GM-CSF or IL-3 showed a 2.5-3.0-fold increase in their capacity to produce leukotriene $\mathrm{C}_{4}\left(\mathrm{LTC}_{4}\right)$, although the mechanism of this increase has not been delineated $(12$,

1. Abbreviations used in this paper: FLAP, 5-lipoxygenase-activating protein; 5-HETE, 5(S)-hydroxy-6-trans-8,11,14-cis-eicosatetraenoic acid; LTA $_{4}$, leukotriene $\mathrm{A}_{4}, 5(S) 5,6$-oxido-7,9-trans-11,14-cis-eicosatetraenoic acid; $\mathrm{LTB}_{4}$, leukotriene $\mathrm{B}_{4}, 5(S), 12(R)$-dihydroxy-6,14cis,8,10-trans-eicosatetraenoic acid; $\mathrm{LTC}_{4}$, leukotriene $\mathrm{C}_{4}, 5(S)$ hydroxy-6(R)-S-glutathionyl-7,9-trans-11,14-cis-eicosatetraenoic acid. 
13). In human neutrophils, eosinophils, and whole blood, priming with GM-CSF or TNF- $\alpha$ for $15-30$ min increases the cells' capacity for synthesis of 5-lipoxygenase products (14$18)$. When conditioned for $4 \mathrm{~h}$ with GM-CSF, human neutrophils have increased levels of 5-lipoxygenase protein, FLAP protein, and FLAP mRNA, but not 5-lipoxygenase mRNA $(19,20)$. Recent work in human neutrophils reported that GM-CSF caused a short-term increase in 5-lipoxygenase activity which is not related to increased 5-lipoxygenase mRNA expression or 5-lipoxygenase protein synthesis (21). In addition, these authors found a more sustained increase in 5-lipoxygenase activity by $18 \mathrm{~h}$, which was associated with the transcriptional activation of the 5-lipoxygenase gene, an increase in 5-lipoxygenase mRNA levels, and new 5-lipoxygenase protein synthesis. Studies by other investigators found that when human monocytes were coincubated with a monoclonal lymphocytic cell line for $10 \mathrm{~min}$, there was an $80 \%$ increase in 5-lipoxygenase activity as reflected by leukotriene $\mathrm{B}_{4}\left(\mathrm{LTB}_{4}\right)$ release (22). Taken together, these studies have been limited either by the short time frame of the investigation or by reliance on cell lines. Studies examining the regulation of the 5-lipoxygenase pathway have been limited by the fact that human cells lose this activity during primary cell cultures. Moreover, these studies have not demonstrated levels of activity in these cells that are comparable with those in primary isolates of inflammatory cells.

We have recently developed an in vitro model using human monocyte-derived macrophages (7a), allowing the study of 5lipoxygenase regulation during long-term culture. Because lymphocytes are potent modulators of immune and inflammatory responses, the goal of this study was to examine the possible role of lymphocytes in regulating expression of the 5-lipoxygenase pathway in monocytes. We examined the effects of lymphocytes on 5-lipoxygenase and FLAP protein and mRNA expression in monocytes and the mechanism of any effect.

\section{Methods}

Cell preparation. Peripheral blood was obtained from normal healthy subjects without known allergy, who had not taken any medications for $2 \mathrm{wk}$ and had not taken aspirin products for $6 \mathrm{wk}$ before blood donation. All subjects were volunteers and had given informed consent to a protocol approved by the Committee on Investigations Involving Human Subjects of the University of California, San Diego.

Monocytes were obtained from peripheral blood by previously described techniques $(7 \mathrm{a}, 23)$. Briefly, blood was collected into acidcitrate-dextrose and red blood cells were allowed to sediment in the presence of dextran. The leukocytes were then collected and washed to remove platelets. Monocytes and lymphocytes were isolated by layering the leukocyte suspension over isotonic Percoll adjusted to a specific gravity of 1.077. Monocytes were further purified by adherence for $50 \mathrm{~min}$ to gelatin-coated plastic flasks, previously incubated with autologous serum, followed by removal of the nonadherent lymphocytes. The lymphocytes were collected and saved. The monocytes were then released by incubation for $15 \mathrm{~min}$ in $5 \mathrm{mM}$ EDTA. Differential cell counts revealed that these preparations were $>95 \%$ monocytes and $>99 \%$ viable. In some studies, T lymphocytes were isolated from collected lymphocytes by $\mathrm{T}$ cell enrichment columns using negative selection (> 96\% CD3 + cells; Human T-Cell Enrichment Column; R \& D Systems, Minneapolis, MN). Cell preparation was performed at room temperature using sterile techniques in a laminar flow hood and endotoxin-free $(<10 \mathrm{pg} / \mathrm{ml})$ solutions. Monocytes and lymphocytes were cultured in RPMI 1640 medium with $10 \%$ au- tologous serum to which $100 \mathrm{U} / \mathrm{ml}$ of penicillin, $100 \mu \mathrm{g} / \mathrm{ml}$ of streptomycin, and $100 \mu \mathrm{g} / \mathrm{ml}$ of gentamicin were added.

Lectin activation of cells. For lectin stimulation studies of both monocytes and lymphocytes, cells were incubated for $4 \mathrm{~d}$ with $10 \mu \mathrm{g} /$ $\mathrm{ml}$ of concanavalin-A and $10 \mu \mathrm{g} / \mathrm{ml}$ of phytohemagglutinin.

Preparation of nonactivated and lectin-activated lymphocyte supernatants. Lymphocytes were incubated for $4 \mathrm{~d}$ without (nonactivated) or with (lectin-activated; L-Stim) lectins. The medium was then collected, spun at $250 \mathrm{~g}$ for $10 \mathrm{~min}$ to remove cells and cellular debris, and the supernatant stored at $4^{\circ} \mathrm{C}$. In some studies, T lymphocytes were used.

Cell disruption. Disrupted cells were prepared by previously described techniques (7a). Briefly, monocytes were gently released from tissue culture plastic with a rubber policeman, the resulting cell suspension was centrifuged, and the cell pellet was disrupted by sonication at $4^{\circ} \mathrm{C}$. Total protein was measured by the Bradford technique (24).

Assay of 5-lipoxygenase activity in intact cells. 5-Lipoxygenase activity was measured in intact cells as previously described (7a). Briefly, activity was measured by collecting and spinning the culture media to collect any nonadherent cells. The resulting cell pellet was resuspended in warmed HBSS and reapplied to the adherent cells. The cells were then stimulated with $1 \mu \mathrm{M} \mathrm{A} 23187$ at $37^{\circ} \mathrm{C}$ for $15 \mathrm{~min}$. The supernatant was collected and lipids were extracted from this supernatant using methanol and chloroform by previously described techniques (25).

Assay of 5-lipoxygenase activity in disrupted cells. 5-Lipoxygenase activity was measured in disrupted cells as previously described (26). Briefly, samples were incubated in a $37^{\circ} \mathrm{C}$ shaking water bath with $2 \mathrm{mM} \mathrm{CaCl}, 2 \mathrm{mM}$ ATP, and $100 \mu \mathrm{M}$ arachidonic acid. After chilling and acidifying the samples, precipitated protein was removed by centrifugation. The supernatants were collected, evaporated to dryness, and residues dissolved in methanol for storage at $-70^{\circ} \mathrm{C}$ until further analysis.

Identification and quantification of lipoxygenase metabolites. Identification and quantification of lipoxygenase products, including 5-HETE and $\mathrm{LTB}_{4}$, were performed on the supernatants from the intact cell and disrupted cell assays by reverse-phase HPLC using previously described techniques (26).

Radiolabeling and assessment of arachidonic acid release. Arachidonic acid uptake, spontaneous release, and stimulated release were assayed using $\left[{ }^{14} \mathrm{C}\right]$ arachidonic acid by previously described techniques (11). Cells were labeled overnight with $0.05 \mu \mathrm{Ci}$ of $\left[{ }^{14} \mathrm{C}\right]$ arachidonic acid and then washed to remove unincorporated label.

Assay of 5-lipoxygenase and FLAP protein and $m R N A$ levels. Immunoblot and Northern blot analyses were performed on total protein and total RNA, respectively, using previously described techniques (7a). Immunoblots were probed with antibodies (generously provided by Dr. Jilly Evans, Merck-Frosst Centre for Therapeutic Research, Pointe Claire-Dorval, Quebec, Canada) that were raised against either recombinant human leukocyte 5-lipoxygenase (1:3,000 dilution) or amino acid residues 41-52 of the human FLAP sequence (1:5,000 dilution). Northern blots were probed with cDNA probes for 5-lipoxygenase (a generous gift of Dr. Richard A. F. Dixon, Merck Sharp and Dohme Research Laboratories, West Point, PA) and FLAP (a generous gift of Dr. Jilly Evans). Bands were quantitated by scanning laser densitometry (LKB Instruments, Inc., Gaithersburg, MD).

Quantification of GM-CSF and IL-3 concentrations. GM-CSF and IL-3 levels were measured using commercially available solid-phase ELISA kits according to the manufacturer's instructions (Quantikine Human GM-CSF or IL-3 Immunoassay; R \& D Systems, Inc.).

Materials. HBSS and RPMI 1640 were obtained from BioWhittaker, Inc. (Walkersville, MD). Penicillin and streptomycin were obtained from the Cell Culture Facility, University of California, San Diego. Gentamicin was obtained from Gemini Bioproducts (Calabasas, CA). Dextran-500 and Percoll were obtained from Pharmacia Fine Chemicals (Piscataway, NJ), A23187 from Calbiochem-Behring Corp. (La Jolla, CA), and redistilled-in-glass grade chromatography 
solvents from Burdick and Jackson Division, Baxter Corp. (Muskegon, MI). 5(S)-HETE was obtained from BIOMOL Research Labs Inc. (Plymouth Meeting, PA). $\mathrm{LTB}_{4}$ and $\mathrm{LTC}_{4}$ were generous gifts of Merck-Frosst Canada (Montreal, Canada). Arachidonic acid was from $\mathrm{Nu}$ Check Prep, Inc. (Elysian, MN). Recombinant human GMCSF, IL-1 $\beta$, IL-2, IL-3, IL-4, IL-6, and TNF- $\alpha$ were obtained from R \& D Systems, Inc. Recombinant human IFN- $\gamma$ and TGF- $\beta_{2}$ were obtained from Genzyme Corp. (Cambridge, MA). Recombinant human IL-10 was obtained from PeproTech, Inc. (Rocky Hill, NJ). Recombinant human leukocyte chemotactic factor was a generous gift of Dr. William Cruikshank of The Pulmonary Center, Boston University School of Medicine (Boston, MA). Human T-Stim with PHA ${ }^{\circledR}$ was obtained from the Collaborative Biomedical Products Division of Collaborative Research (Bedford, MA). All other chemicals were from Sigma Chemical Co. (St. Louis, MO) and were of the finest grade available.

Data analysis. Data are expressed as the mean \pm SEM in all circumstances where mean values are compared. Matched pairs were analyzed with a two-tailed paired $t$ test. Other differences between means were analyzed with a two-tailed unpaired $t$ test. Time course and dose-response studies were analyzed with a repeated measures ANOVA using a Fisher's post-hoc test. Differences were considered significant when $P<0.05$ (27).

\section{Results}

Effect of lymphocytes on monocyte 5-lipoxygenase activity during coculture. Previous studies in our laboratory have shown that 5-lipoxygenase and FLAP expression decrease in culture over time (7a). For these studies, monocytes were cultured initially alone for $4 \mathrm{~d}$. The monocytes were then cocultured an additional $2 \mathrm{~d}$ with autologous lymphocytes, either in the presence or absence of lectins, at concentrations of one million monocytes and eight million lymphocytes per milliliter. This ratio approximates that found in normal peripheral blood.

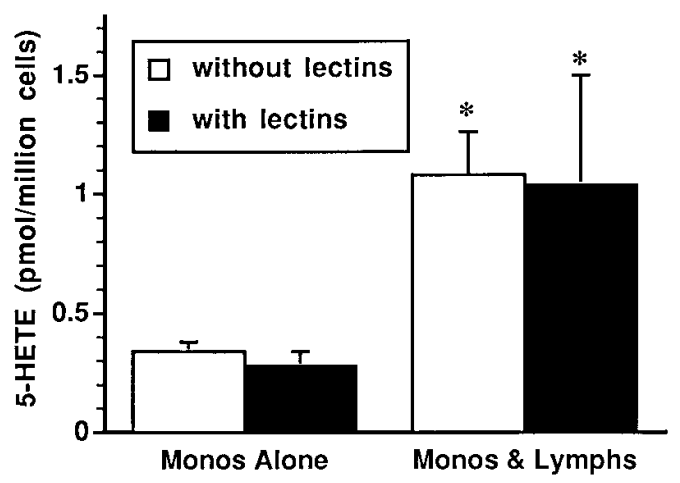

Figure 1. Effects of lymphocytes on monocyte 5-lipoxygenase activity during coculture. Monocytes and lymphocytes were isolated from normal volunteers by density gradient centrifugation and adherence techniques. Monocytes were cultured for $4 \mathrm{~d}$ in RPMI 1640 with $10 \%$ autologous serum. Lymphocytes, from the same donor, were incubated for $4 \mathrm{~d}$ with or without lectins. Monocytes were then cultured for an additional $2 \mathrm{~d}$ alone or cocultured with lymphocytes at a ratio of one monocyte to eight lymphocytes, with or without lectins. The cells were then washed with HBSS and stimulated with $1 \mu \mathrm{M}$ A23187 for $15 \mathrm{~min}$ at $37^{\circ} \mathrm{C}$. Supernatants were collected, extracted, and analyzed for lipoxygenase products by reverse-phase HPLC and ultraviolet spectroscopy. Data are expressed as picomoles of 5-HETE per $10^{6}$ cells. 5-HETE release was significantly increased by coculture with lymphocytes $(* P<0.05)$ and lectins had no further effect. Data represent the mean \pm SEM of at least three experiments.
A23187-stimulated whole cell activity was then assessed. The addition of lymphocytes resulted in a significant increase in stimulated monocyte 5-lipoxygenase activity, as reflected by a threefold increase in 5-HETE $(P<0.05)$ (Fig. 1) and $\mathrm{LTB}_{4}$ release $(1.7 \pm 0.3$ vs. $0.6 \pm 0.1 \mathrm{pmol} / \mathrm{million}$ cells, cocultured vs. control monocytes; $n \geq 3 ; P<0.05$ ). Lectins had no effect on baseline monocyte 5-lipoxygenase activity or on the increase in activity observed with lymphocyte coculture $(P=$ NS) (Fig. 1$)$. When lymphocytes were stimulated with A23187 in the absence of monocytes, no 5-lipoxygenase activity was detected. There was no detectable $\mathrm{LTC}_{4}$ in any intact cell assays.

Effect of lymphocyte supernatants on monocyte 5-lipoxygenase activity. To determine if the increase in monocyte 5-lipoxygenase activity seen during coculture with lymphocytes was due to a soluble factor(s), monocytes were cultured alone for $4 \mathrm{~d}$ and then cultured an additional $2 \mathrm{~d}$ with $10 \%$ supernatant from lymphocytes, conditioned with or without lectins. A23187stimulated whole cell activity was then assessed. The supernatant from nonactivated lymphocytes had no effect on monocyte 5-lipoxygenase activity, as reflected by the release of both 5-HETE $(P=\mathrm{NS})$ (Fig. 2$)$ and $\mathrm{LTB}_{4}(0.5 \pm 0.2$ vs. $0.4 \pm 0.1$ $\mathrm{pmol} / \mathrm{million}$ cells, conditioned vs. control; $n \geq 3 ; P=\mathrm{NS})$. However, the supernatant from lectin-activated lymphocytes caused a significant increase in monocyte 5-lipoxygenase activity, as reflected by a fourfold increase in 5-HETE $(P<0.05)$ (Fig. 2) and a sixfold increase in $\mathrm{LTB}_{4}$ release $(2.3 \pm 0.9$ vs. $0.4 \pm 0.1 \mathrm{pmol} / \mathrm{million}$ cells, conditioned vs. control; $n \geq 5 ; P<$ 0.05). When the same experiments were carried out in cells cultured in the usual culture medium, but in which $10 \%$ autologous serum had neither been heat treated nor sterile filtered, the supernatant from lectin-activated lymphocytes still increased monocyte 5-lipoxygenase activity (2.9 \pm 0.2 vs. $0.2 \pm 0.1$ pmol of 5-HETE per million cells, conditioned vs. control monocytes; $n \geq 3 ; P<0.05)$.

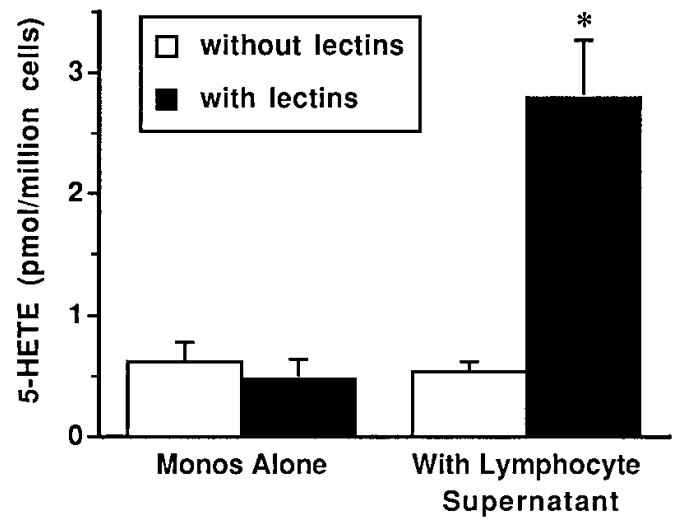

Figure 2. Effects of lymphocyte supernatants on monocyte 5-lipoxygenase activity. Monocytes and lymphocytes were prepared as in Fig. 1. Conditioned supernatants were collected from lymphocyte cultures with or without lectins and were added to monocyte cultures at a final concentration of $10 \%(\mathrm{vol} / \mathrm{vol})$. Monocytes were then cultured for an additional $2 \mathrm{~d}$ alone, with lectins, or with supernatant from either nonactivated or lectin-activated lymphocytes. Stimulated 5-lipoxygenase activity was assessed as in Fig. 1. Data are expressed as picomoles of 5-HETE per $10^{6}$ cells. 5-HETE release was significantly increased only by coculture with supernatants from lectin-activated lymphocytes $\left({ }^{*} P<0.05\right)$. Data represent the mean \pm SEM of at least three experiments. 
Effect of activated T lymphocyte supernatants on monocyte 5-lipoxygenase activity. To determine the type of lymphocyte that was primarily responsible for this increased activity, monocytes were cultured alone for $4 \mathrm{~d}$ and then cultured an additional $2 \mathrm{~d}$ with $10 \%$ supernatant from lectin-activated $\mathrm{T}$ lymphocytes or with $10 \%$ Human T-Stim with PHA $^{\circledR}$ (purchased supernatant from lectin-activated T lymphocytes). Similar results were obtained $(0.2 \pm 0.1$ vs. $2.5 \pm 0.6$ vs. $4.2 \pm 0.8$ pmol of 5-HETE per million cells, control vs. lectin-activated T lymphocyte supernatant conditioned monocytes vs. Human T-Stim with PHA ${ }^{\circledR}$ conditioned monocytes; $n \geq 3 ; P<0.05$ ). There was no significant difference in product release between monocytes conditioned with lectin-activated lymphocyte supernatant, lectin-activated $\mathrm{T}$ lymphocyte supernatant, and $\mathrm{Hu}-$ man T-Stim with $\mathrm{PHA}^{\circledR}(P=\mathrm{NS})$. Similar results were obtained for $\mathrm{LTB}_{4}$ (data not shown).

Time course of effect of lectin-activated lymphocyte supernatant on monocyte 5-lipoxygenase activity. Monocytes were cultured alone for $4 \mathrm{~d}$, and then cultured with $10 \%$ supernatant from lectin-activated lymphocytes for various lengths of time. A23187-stimulated whole cell activity was then assessed. Before conditioning, monocytes released 5.1 \pm 2.2 pmol of 5-HETE and 4.2 \pm 0.8 pmol of $\mathrm{LTB}_{4}$ per million cells $(n \geq 3)$. Paired analysis was performed on matched controls for each subject. There was an initial increase in the capacity for 5-HETE release at $15 \mathrm{~min}$ of conditioning with the supernatant from lectin-activated lymphocytes, but the capacity for 5-HETE release returned to baseline by $1 \mathrm{~h}$ (Fig. 3). Capacity for 5-HETE release in conditioned cells increased again at $8 \mathrm{~h}$, peaked at $2 \mathrm{~d}$ (eightfold increase), and this increase persisted through $4 \mathrm{~d}$ of conditioning. Similar results were obtained for $\mathrm{LTB}_{4}$ release $(195 \pm 14 \%, 104 \pm 1 \%, 166 \pm 8 \%, 451 \pm 60 \%$,

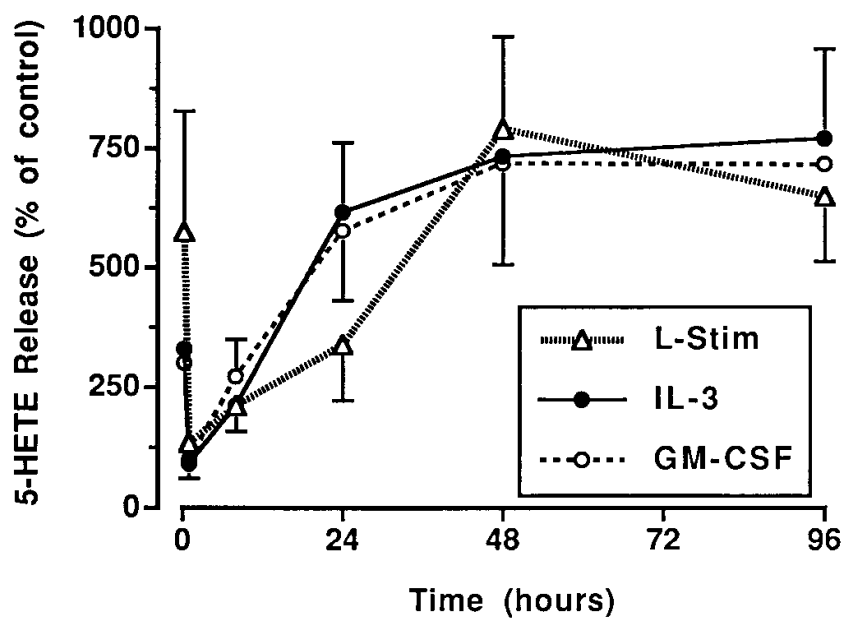

Figure 3. Time course of the effect of lectin-activated lymphocyte supernatant, GM-CSF, and IL-3 on monocyte 5-lipoxygenase activity. Lectin-activated lymphocyte supernatants were prepared as in Fig. 2. Monocytes were prepared as in Fig. 1. Monocytes were then cultured with $10 \%$ lectin-activated lymphocyte supernatants, GM-CSF, or IL-3 for up to $96 \mathrm{~h}$. At various time points, stimulated 5-lipoxygenase activity was assessed as in Fig. 1. Data are expressed as a percentage of control of stimulated 5-HETE release. 5-HETE release was significantly increased compared with control after 15 min conditioning with activated lymphocyte supernatant and in all conditioning studies at 2 and $4 \mathrm{~d}$. Data represent the mean \pm SEM of at least three experiments.
$575 \pm 225 \%$, and $403 \pm 57 \%$, percent change in $\mathrm{LTB}_{4}$ release from control at $15 \mathrm{~min}, 1 \mathrm{~h}, 8 \mathrm{~h}, 1 \mathrm{~d}, 2 \mathrm{~d}$, and $4 \mathrm{~d}$, respectively). Compared with percent change in 5-HETE and $\mathrm{LTB}_{4}$ release between control and conditioned cells at $1 \mathrm{~h}$, the increases at $15 \mathrm{~min}, 2 \mathrm{~d}$, and $4 \mathrm{~d}$ were significant $(P<0.05)$.

Effect of lymphocyte supernatant on monocyte arachidonic acid release. To determine if a change in arachidonic acid release contributed to the increased synthesis of 5-lipoxygenase products seen in the conditioned monocytes, monocytes were cultured alone for $4 \mathrm{~d}$ and then cultured an additional $2 \mathrm{~d}$ with $10 \%$ supernatant from lectin-activated lymphocytes. The cells were labeled overnight with $\left[{ }^{14} \mathrm{C}\right]$ arachidonic acid, during the last day of conditioning. When compared with controls, conditioned monocytes did not release larger quantities of label after $15 \mathrm{~min}$ of stimulation with $1 \mu \mathrm{M}$ A23187 $(9.3 \pm 0.2 \%$ vs. $4.0 \pm 0.2 \%$ stimulated release of label for control vs. conditioned cells, expressed as a percentage of the total label incorporated).

Effect of lymphocyte supernatant on monocyte 5-lipoxygenase activity in disrupted cells. To determine if an increase in 5-lipoxygenase enzymatic activity was responsible for the increased 5-lipoxygenase activity seen in whole, conditioned monocytes, assays of 5-lipoxygenase activity were performed in disrupted cells. Monocytes were cultured alone for $4 \mathrm{~d}$ and then cultured with or without $10 \%$ supernatant from lectinactivated lymphocytes for an additional $2 \mathrm{~d}$. Disrupted cell fractions were then assayed for 5-lipoxygenase and $\mathrm{LTA}_{4}$ hydrolase activities. 5-Lipoxygenase activity was increased in disrupted cell fractions from conditioned monocytes when compared with controls $(240.9 \pm 36.1 \%, 5$-HETE production as a percentage of control, $P<0.05)$. There was no difference in LTA $_{4}$ hydrolase activity $\left(109.6 \pm 12.3 \%, \mathrm{LTB}_{4}\right.$ production as a percentage of control, $P=$ NS).

Effect of lymphocyte supernatant on monocyte 5-lipoxygenase and FLAP protein levels. To determine if the ability of lectin-activated lymphocyte supernatant to increase 5-lipoxygenase activity resulted from an increase in immunoreactive 5-lipoxygenase and FLAP, monocytes were cultured alone for $4 \mathrm{~d}$ and then cultured with or without $10 \%$ supernatant from lectin-activated lymphocytes for an additional $2 \mathrm{~d}$. Immunoblotting of these cells revealed a 2.4-fold increase in 5-lipoxygenase and a 3.2-fold increase in FLAP immunoreactive protein when disrupted cell fractions from conditioned cells were compared with those from control cells (Fig. 4).

Effect of lymphocyte supernatant on monocyte 5-lipoxygenase and FLAP mRNA levels. To determine the effect of lectinactivated lymphocyte supernatant on mRNA encoding for 5-lipoxygenase and FLAP, monocytes were cultured alone for $4 \mathrm{~d}$ and then cultured with or without $10 \%$ supernatant from

\section{Control L-Stim Figure 4. Immunoblot} analysis for 5-lipoxygenase

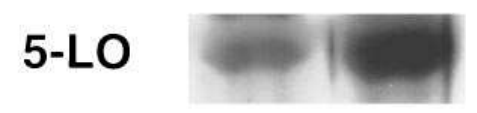
and FLAP in disrupted monocytes. Monocytes and lectin-activated lympho-

FLAP $=\begin{aligned} & \text { cyte supernatants were pre- } \\ & \text { pared as in Fig. 2. Mono- }\end{aligned}$ cytes were cultured without (Control) or with $10 \%$ lectin-activated lymphocyte supernatants (L-Stim) for $2 \mathrm{~d}$. Immunoblots for 5-lipoxygenase (top) or FLAP (bottom) were performed on disrupted cell fractions, comparing control cells (left) and conditioned cells (right). 


\section{Control L-Stim}

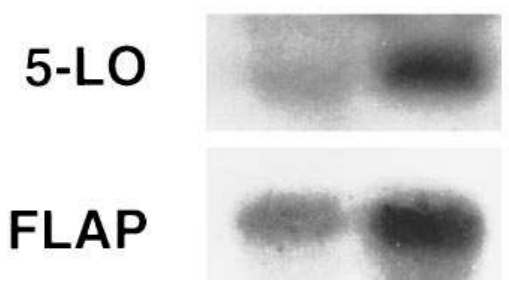

Figure 5. Northern blot for 5-lipoxygenase and FLAP. Monocytes and lectin-activated lymphocyte supernatants were prepared as in Fig. 2. Monocytes were cultured without (Control) or with $10 \%$ lectin-activated lymphocyte supernatants $(L-S t i m)$ for $2 \mathrm{~d}$. Total RNA was extracted and subjected to standard Northern blot analysis. Autoradiographs of a representative blot probed for 5lipoxygenase (top) and FLAP (bottom), comparing control (left) and conditioned (right) cells.

lectin-activated lymphocytes for an additional $2 \mathrm{~d}$. Northern blot analysis of total RNA showed a 3.4-fold increase in 5-lipoxygenase mRNA and a 1.9-fold increase in FLAP mRNA for lectin-activated versus control monocytes (Fig. 5).

Effect of cytokines on monocyte 5-lipoxygenase activity. To study the effects of cytokines, an abbreviated protocol was developed. Monocytes were cultured alone for $1 \mathrm{~d}$ and then conditioned for an additional day with $10 \%$ supernatant from lectin-activated lymphocytes, or with various cytokines known to be released by activated lymphocytes. A23187-stimulated whole cell activity was then assessed. Conditioning with $10 \%$ lectin-activated lymphocyte supernatant caused a fourfold increase in 5-HETE and $\mathrm{LTB}_{4}$ release compared with control $(P<0.05)$ (Table I). Similarly, conditioning with $10 \mathrm{ng} / \mathrm{ml}$ of GM-CSF or $10 \mathrm{ng} / \mathrm{ml}$ of IL-3 also caused an approximately fourfold increase in 5-HETE and $\mathrm{LTB}_{4}$ release $(P<0.05)$. The combination of $10 \mathrm{ng} / \mathrm{ml}$ of GM-CSF and $10 \mathrm{ng} / \mathrm{ml}$ of IL-3 re-

Table I. Effects of Cytokines on Monocyte 5-Lipoxygenase Activity

\begin{tabular}{lcc}
\hline \multicolumn{1}{c}{ Condition } & 5-HETE & LTB $_{4}$ \\
\hline Control & $5.7 \pm 0.8$ & $3.3 \pm 0.7$ \\
L-Stim & $22.6 \pm 5.4^{*}$ & $11.8 \pm 2.3^{*}$ \\
GM-CSF & $21.0 \pm 3.9^{*}$ & $15.0 \pm 2.0^{*}$ \\
IFN- $\gamma$ & $10.5 \pm 0.7$ & $4.0 \pm 1.6$ \\
IL-1 & $5.8 \pm 1.4$ & $1.5 \pm 1.1$ \\
IL-2 & $8.0 \pm 2.1$ & $4.1 \pm 2.3$ \\
IL-3 & $26.3 \pm 7.1^{*}$ & $17.0 \pm 2.7^{*}$ \\
IL-4 & $7.3 \pm 1.7$ & $2.6 \pm 0.6$ \\
IL-6 & $4.7 \pm 1.1$ & $1.9 \pm 0.3$ \\
IL-10 & $5.0 \pm 2.3$ & $3.2 \pm 1.6$ \\
LCF & $2.8 \pm 0.2$ & $1.9 \pm 0.6$ \\
TGF- $\beta$ & $3.1 \pm 0.0$ & $2.2 \pm 0.1$ \\
TNF- $\alpha$ & $2.8 \pm 0.7$ & $1.9 \pm 0.5$ \\
GM-CSF/IL-3 & $27.5 \pm 3.5^{*}$ & $17.5 \pm 0.7^{*}$ \\
\end{tabular}

Monocytes and lectin-activated lymphocyte supernatants were prepared as in Fig. 2. Monocytes were then conditioned for an additional day with lectin-activated lymphocyte supernatants $(L-S t i m)$ or a variety of cytokines, including the combination of GM-CSF and IL-3 (GM-CSF/IL-3). After conditioning, stimulated 5-lipoxygenase activity was assessed as in Fig. 1. Data are expressed as mean picomoles of product released per $10^{6}$ cells \pm SEM of at least three experiments, for 5-HETE release and $\mathrm{LTB}_{4}$ release. $*$ Results which are significantly increased compared with control $(P<0.05)$ sulted in no additional 5-HETE or $\mathrm{LTB}_{4}$ release, compared with either GM-CSF or IL-3 alone ( $P=\mathrm{NS})$. There was no significant change in 5-HETE or $\mathrm{LTB}_{4}$ release compared with control when monocytes were conditioned with IFN- $\gamma$ at 40 $\mathrm{ng} / \mathrm{ml}$, with IL- $1 \beta$ at $1 \mathrm{ng} / \mathrm{ml}$, or with either IL-2, IL-4, IL-6, IL10 , leukocyte chemotactic factor, TGF- $\beta$, or TNF- $\alpha$, each at 10 $\mathrm{ng} / \mathrm{ml}(P=\mathrm{NS}$ in all cases $)$.

Time course of the effect of GM-CSF and IL-3 on monocyte 5-lipoxygenase activity. Monocytes were cultured alone for $1 \mathrm{~d}$ and then conditioned with either GM-CSF or IL-3 for various lengths of time. A23187-stimulated whole cell activity was then assessed. Before conditioning, monocytes released 5.3 \pm 0.3 pmol of 5-HETE per million cells $(n=3)$. Paired analysis was performed on matched controls for each subject. Time course studies showed similar results for both GM-CSF and for IL-3. There was an initial increase in the capacity for 5-HETE release at $15 \mathrm{~min}$ of conditioning, but this release returned to baseline by $1 \mathrm{~h}$ (Fig. 3). The capacity for 5-HETE release again increased at $8 \mathrm{~h}$, increased sixfold by $1 \mathrm{~d}$ and persisted up to $4 \mathrm{~d}$ of conditioning. Compared with 5-HETE release at $1 \mathrm{~h}$, the increases at 2 and $4 \mathrm{~d}$ were significant $(P<0.05)$. Similar results were obtained for $\mathrm{LTB}_{4}$ release (data not shown).

Dose-response of the effect of GM-CSF and IL-3 on monocyte 5-lipoxygenase activity. Monocytes were cultured alone for $1 \mathrm{~d}$ and then conditioned with either GM-CSF or IL-3 for an additional day at various concentrations. A23187-stimulated whole cell activity was then assessed. Dose-response studies

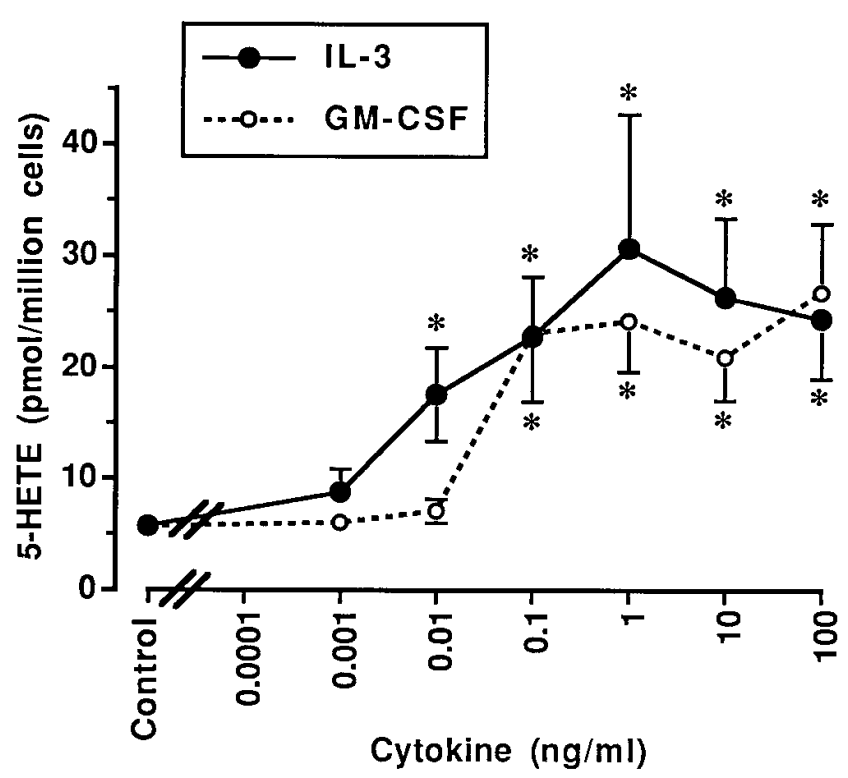

Figure 6. Dose-response of GM-CSF and IL-3 on 5-lipoxygenase activity in monocytes. Monocytes were prepared as in Fig. 1 and cultured for $1 \mathrm{~d}$. These cells were then conditioned with up to $100 \mathrm{ng} / \mathrm{ml}$ of GM-CSF or IL-3 for $1 \mathrm{~d}$. After conditioning, stimulated 5-lipoxygenase activity was assessed as in Fig. 1. Data are expressed as picomoles of 5-HETE per $10^{6}$ cells; data represent the mean \pm SEM of at least three experiments. For GM-CSF dose-response, there was a significant increase in 5-HETE release at $100 \mathrm{pg} / \mathrm{ml}$ compared with control, which then largely remained stable at higher dosages; the $\mathrm{ED}_{50}$ was calculated as $51 \mathrm{pg} / \mathrm{ml}$. For IL-3 dose-response, there was a significant increase in 5-HETE release at $10 \mathrm{pg} / \mathrm{ml}$, which continued to show some increase through $100 \mathrm{ng} / \mathrm{ml}$; the $\mathrm{ED}_{50}$ was calculated as $18 \mathrm{pg} / \mathrm{ml} . * P<0.05$. 

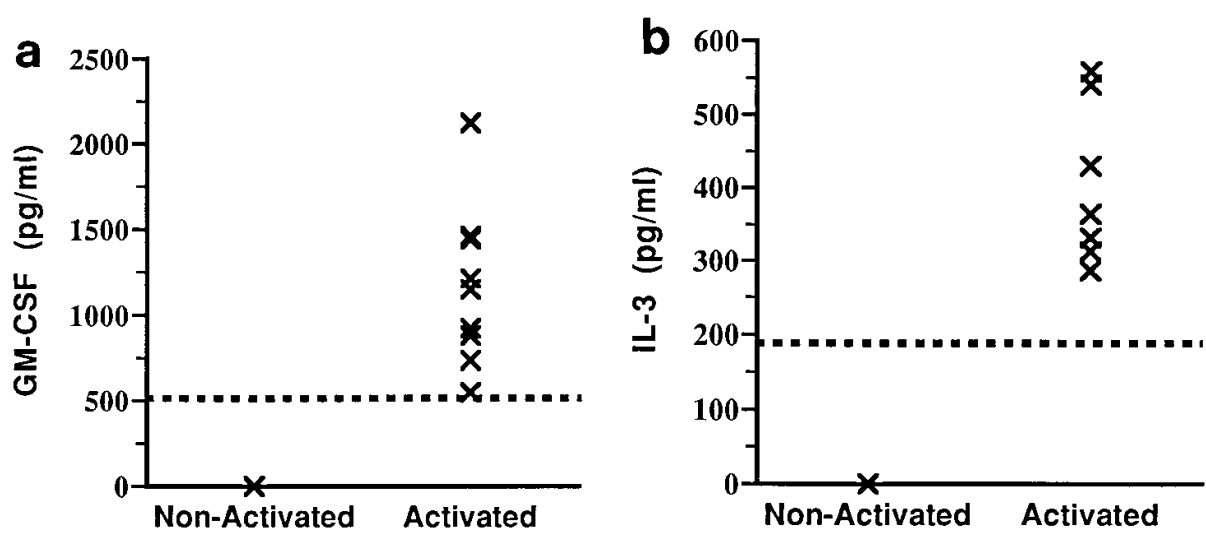

Figure 7. Concentrations of GM-CSF and IL-3 in nonactivated and lectin-activated lymphocyte supernatants. Lymphocyte supernatants were prepared as in Fig. 2. Levels of GM-CSF and IL-3 were measured in supernatants from nonactivated and activated lymphocytes by solid-phase ELISA $(n \geq 8)$. Results are expressed as picograms per milliliter for GM-CSF ( $a$ ) and IL-3 (b). The $\mathrm{ED}_{50}$, correcting for $10 \%$ dilution in culture media, is denoted by horizontal dashed lines. All lectin-activated lymphocyte supernatants studied had concentrations of both GM-CSF and IL-3 above the threshold levels for increasing 5-lipoxygenase activity in monocytes. for GM-CSF revealed no significant differences in stimulated 5 -HETE release between control, $1 \mathrm{pg} / \mathrm{ml}$, and $10 \mathrm{pg} / \mathrm{ml}$ of GM-CSF (Fig. 6). At $100 \mathrm{pg} / \mathrm{ml}$, there was a fourfold increase in 5-HETE release $(P<0.05)$. This increase persisted without significant change up to $100 \mathrm{ng} / \mathrm{ml}$ of GM-CSF. Similar results were obtained for $\mathrm{LTB}_{4}$ release (data not shown). The calculated $\mathrm{ED}_{50}$ for GM-CSF was $51 \mathrm{pg} / \mathrm{ml}$. Dose-response studies for IL-3 revealed no significant difference in 5-HETE release between control and $1.0 \mathrm{pg} / \mathrm{ml}$ (Fig. 6). At $10 \mathrm{pg} / \mathrm{ml}$, there was a 3.1-fold increase in 5-HETE release which further increased 4-fold at $100 \mathrm{ng} / \mathrm{ml}$ of IL-3. Compared with control, a significant increase in 5-HETE release was first found at $10 \mathrm{pg} / \mathrm{ml}$ $(P<0.05)$. Similar results were obtained for $\mathrm{LTB}_{4}$ release (data not shown). The calculated $\mathrm{ED}_{50}$ for IL-3 was $18 \mathrm{pg} / \mathrm{ml}$.

GM-CSF and IL-3 levels in supernatants from nonactivated and lectin-activated lymphocytes. To determine if either GM-CSF or IL-3 was present in sufficient concentrations in lymphocyte supernatants to induce an increase in monocyte 5-lipoxygenase activity, concentrations of these cytokines were measured. GM-CSF and IL-3 were not detectable in supernatants from lymphocytes not activated with lectins (nonactivated lymphocyte supernatant) (sensitivity of the assay 1.5 and $7.4 \mathrm{pg} / \mathrm{ml}$, respectively) (Fig. 7, $a$ and $b$ ). Cytokine levels in supernatants from lectin-activated lymphocytes were $1,163 \pm 157.9 \mathrm{pg} / \mathrm{ml}$ for GM-CSF and $271.2 \pm 68.2 \mathrm{pg} / \mathrm{ml}$ for IL-3. In all supernatants from lectin-activated lymphocytes, both cytokines were present at levels above the threshold levels of the cytokines needed to increase 5-HETE release in monocytes $(510 \mathrm{pg} / \mathrm{ml}$ of GM-CSF and $180 \mathrm{pg} / \mathrm{ml}$ of IL-3), after accounting for the 10 -fold dilution that occurs when adding $10 \%$ lectin-activated lymphocyte supernatant to monocyte culture.

Antibodies to GM-CSF and IL-3 block the effect of lectinactivated lymphocyte supernatant on monocyte 5-lipoxygenase activity. Monocytes were cultured alone for $4 \mathrm{~d}$ and then cultured for an additional $2 \mathrm{~d}$ with or without $10 \%$ supernatant from lectin-activated lymphocytes. Lectin-activated lymphocyte supernatants were preincubated alone or with $50 \mu \mathrm{g} / \mathrm{ml}$ of antibodies to GM-CSF and/or IL-3 (anti-human GM-CSF or IL-3 neutralizing antibody; R \& D Systems, Inc.) for $30 \mathrm{~min}$ before adding the supernatants to the monocytes. A23187stimulated whole cell activity was then assessed. 5-HETE release was significantly increased compared with control in monocytes conditioned with lectin-activated lymphocyte supernatant alone, as in experiments reported above $(P<0.05)$
(Fig. 8). Likewise, 5-HETE release was significantly increased compared with control in monocytes conditioned with lectinactivated lymphocyte supernatant treated with antibodies to either GM-CSF or IL-3 $(P<0.05)$ (Fig. 8). However, when monocytes were cultured with lectin-activated lymphocyte supernatant treated with antibodies to both GM-CSF and IL-3, this effect of lectin-activated lymphocyte supernatant was significantly inhibited and therefore 5-HETE release was not significantly different compared with control $(P=$ NS) (Fig. 8). Similar results were obtained for $\mathrm{LTB}_{4}$ release (data not shown).

\section{Discussion}

Our studies demonstrate that lymphocytes, cocultured with monocytes over a period of several days, induce a large increase in the capacity of monocytes to synthesize 5-lipoxygenase products. Increases in monocyte 5-lipoxygenase activity are also observed when the monocytes are conditioned with supernatant from lectin-activated lymphocytes, but are not found when monocytes are conditioned with supernatant from nonactivated lymphocytes. This increase in activity appears to be primarily due to the influence of activated $\mathrm{T}$ lymphocytes. After an initial, transient increase in monocyte 5-lipoxygenase activity, there is a gradual increase over $2 \mathrm{~d}$. We also find that lymphocytes in coculture with monocytes do not require lectin activation to cause this effect. The activated lymphocytes thus release a soluble factor(s) into their supernatant that increases the capacity of monocytes to synthesize 5-lipoxygenase products. This increase in activity is not associated with increased availability of arachidonic acid. Rather, this change in activity is associated with increases in both protein and mRNA for 5-lipoxygenase and FLAP. Because of limits in the quantity of primary monocytes, we have been unable to determine if changes in mRNA represent regulation at the transcriptional or posttranscriptional level.

We further report that either GM-CSF or IL-3 causes similar increases in the capacity of monocytes to synthesize 5-lipoxygenase products and does so over a similar time course, when compared with lectin-activated lymphocyte supernatant. GM-CSF and IL-3 are both present in the supernatants from lectin-activated lymphocytes in sufficient quantities to account for the supernatant effects on 5-lipoxygenase activ- 


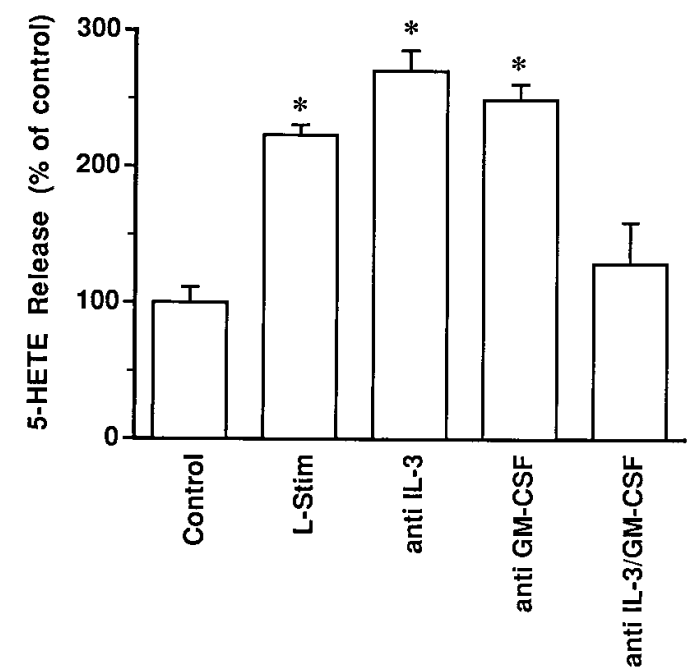

Figure 8. Effects of anti-GM-CSF and anti-IL-3 antibodies on lectinactivated lymphocyte supernatant-mediated increase in 5-lipoxygenase activity. Monocytes and lectin-activated lymphocyte supernatants were prepared as in Fig. 2. The cells were then conditioned for $2 \mathrm{~d}$ with $10 \%$ lectin-activated lymphocyte supernatant $(\mathrm{L}$-Stim $)$ in the presence or absence of neutralizing antibody to GM-CSF, IL-3, or both. After conditioning, stimulated 5-lipoxygenase activity was assessed as in Fig. 1. Data are expressed as a percentage of control of stimulated 5-HETE release. 5-HETE release was significantly increased in conditioned cells compared with control in all cases $(* P<$ $0.05)$, except when conditioned with lectin-activated lymphocyte supernatant in the presence of neutralizing antibodies to both GM-CSF and IL-3. Data represent the mean \pm SD of three experiments.

ity, but are not found in the supernatant from nonactivated lymphocytes. Antibodies to both GM-CSF and IL-3 block the effect of activated lymphocyte supernatant on monocyte 5-lipoxygenase activity. Thus, the increase in the capacity of monocytes to release 5-lipoxygenase products induced by activated lymphocytes is predominantly due to the release of the cytokines GM-CSF and IL-3 from lymphocytes.

The 5-lipoxygenase (28) and FLAP (29) genes have been cloned and several kilobases of their $5^{\prime}$ untranslated regions have been sequenced. Nucleic acid sequence analysis suggests that the 5-lipoxygenase promoter region is most consistent with that of a housekeeping gene and, therefore, 5-lipoxygenase might be constitutively expressed in human cells (30). The FLAP promoter sequence suggests that FLAP expression is regulated and that this protein is not constitutively expressed (29). Studies in conditioned HL-60 cells and in GM-CSF-conditioned human neutrophils have not demonstrated regulation of 5-lipoxygenase mRNA expression $(9,19)$. Many previous studies have addressed the regulation of 5-lipoxygenase activity and, depending on the cell type and the duration of study, have reported changes at the level of arachidonic acid release (17, 31-33), 5-lipoxygenase $(9,19,21,34)$, or FLAP $(10,20)$. In our model of primary cell isolates conditioned with lectin-activated lymphocyte supernatant, we have found regulation of 5-lipoxygenase activity at the level of protein and mRNA expression of both 5-lipoxygenase and FLAP, but found no increase in arachidonic acid release. This is in keeping with previous studies showing increased expression of both proteins in alveolar macrophages, as compared with peripheral blood monocytes $(2,7)$. Our findings are also consistent with the available data on the distribution of 5-lipoxygenase, suggesting that its tissue expression in humans is highly specific and regulated. Our data are consistent with the work of others examining differentiation of HL-60 cells, showing regulation of both 5-lipoxygenase and FLAP (8). Finally, our results are consistent with recent work using run-on analysis in human neutrophils conditioned with GM-CSF, which has provided direct evidence that transcriptional activity of the 5-lipoxygenase gene can be enhanced (21). However, further work will be required to reconcile the various studies showing regulated expression of 5-lipoxygenase with the available DNA sequence data on the 5-lipoxygenase promoter, which suggest that this protein should be constitutively expressed.

We have screened various cytokines known to be released by activated lymphocytes. Compared with the effect of activated-lymphocyte supernatant, we found that either GM-CSF or IL-3 causes similar increases in the capacity of monocytes to release the 5-lipoxygenase products, 5-HETE and $\mathrm{LTB}_{4}$. There was no effect on the capacity of monocytes for 5-lipoxygenation by a wide range of other cytokines, including the cytokines INF- $\gamma$, TGF- $\beta$, and TNF- $\alpha$, which in other systems influence the 5-lipoxygenase pathway $(9,11,15,18)$. GM-CSF $(12,14-21)$ and IL-3 (13) have been reported previously in other systems to have regulatory effects on the 5-lipoxygenase pathway. We found no additive effect of GM-CSF and IL-3 together. These results are consistent with current knowledge about monocytes and about receptors for the cytokines GMCSF and IL-3. When monocytes are cultured over days with either GM-CSF or IL-3, there are increases in cell survival, binding to plastic, and cytotoxic activities (35-37). When monocytes are cultured for $2 \mathrm{wk}$, either GM-CSF or IL-3 facilitates the long-term maturation of monocytes into macrophages, augments their capacity to bind endotoxin, and elevates the release of cytokines involved in inflammatory and granulomatous reactions (38). GM-CSF, IL-3, and IL-5 share a common $\beta$ subunit in their cell surface receptor while having unique $\alpha$ subunits. Since both the $\alpha$ and $\beta$ subunits are required for ligand binding, there can be competition between the cytokines for the $\beta$ subunit, and when all the $\beta$ subunits have been occupied, there is little or no additive effect of an additional cytokine (39). We did not evaluate IL-5 because there is not an $\alpha$ subunit for IL-5 present on human monocytes and, therefore, IL-5 has been shown to have no effect on these cells (40).

Previous work in the myeloid precursor cell line HL-60 had suggested that heat treatment of serum, by activating latent TGF- $\beta$, plays an important role in the regulation of 5-lipoxygenase activity during culture (9). We were unable to address this directly in our system, as our cells required serum for longterm culture. However, we were able to address this issue indirectly. In our cell system, heat treatment of serum, known to activate TGF- $\beta$ (41), had no effect on the increased 5-lipoxygenase activity observed in conditioned monocytes. The lack of influence of heat-treated serum seen in our study may reflect the presence of active TGF- $\beta$ in the activated lymphocyte supernatant or may be due to differences between monocytederived macrophages and DMSO-differentiated HL-60 cells.

Lymphocytes cocultured with monocytes or lymphocytes activated by lectins induce increases in the capacity of monocytes to synthesize 5-lipoxygenase products. However, lymphocytes cultured alone and not activated lack this ability. This would suggest that during coculture monocytes activate lym- 
phocytes to release GM-CSF and IL-3. This is in keeping with extensive previous work showing that monocytes play an important role in the complex regulation of lymphocyte activation (42). Although studies have begun to elucidate the regulation of GM-CSF expression in lymphocytes, the results to date are not sufficient to fully explain our observations with monocyte-lymphocyte coculture (43). Further delineation of this interaction was outside the scope of the present investigation.

In summary, we report a new monocyte-lymphocyte interaction in the regulation of the inflammatory process. Exposure of human monocytes to either lymphocytes or to the supernatant from lectin-activated lymphocytes causes an increase in the capacity of monocytes to release 5-lipoxygenase products. This effect is due, at least in part, to increased expression of 5-lipoxygenase and FLAP, via the release of GM-CSF and IL-3. Thus, the expression of 5-lipoxygenase and FLAP in human monocytes can be controlled by specific cytokines. GM-CSF is increased in the serum of psoriatic patients and increased locally in a wide range of inflammatory diseases associated with leukotrienes, including asthma, psoriasis, allergen-challenged cutaneous late-phase reactions, and idiopathic pulmonary fibrosis (44-49). Therefore, our results may reflect a clinically important means whereby the 5-lipoxygenase pathway is regulated in human disease.

\section{Acknowledgments}

This work was supported in part by a grant from the National Heart, Lung, and Blood Institute of the National Institutes of Health (HL01888), a grant from the Merit Review Board of the Department of Veterans Affairs, and a grant from Glaxo Wellcome. Dr. Ring was supported by a training fellowship award from the American Lung Association of California and a Physician Scientist Award from the National Institutes of Health. Dr. Riddick was supported by a Faculty Development Grant from the Robert Wood Johnson Foundation. Dr. Munafo was supported by a training fellowship award from the Tobacco-Related Disease Research Program of the University of California and a training fellowship award from the American Lung Association. Dr. Bigby was supported by a Career Investigator Award from the American Lung Association.

\section{References}

1. Lewis, R.A., K.F. Austen, and R.J. Soberman. 1990. Leukotrienes and other products of the 5-lipoxygenase pathway. Biochemistry and relation to pathobiology in human diseases. N. Engl. J. Med. 323:645-655.

2. Pueringer, R.J., C.C. Bahns, and G.W. Hunninghake. 1992. Alveolar macrophages have greater amounts of the enzyme 5-lipoxygenase than do monocytes. J. Appl. Physiol. 73:781-786.

3. Woods, J.W., J.F. Evans, D. Ethier, S. Scott, P.J. Vickers, L. Hearn, J.A. Heibein, S. Charleson, and I.I. Singer. 1993. 5-lipoxygenase and 5-lipoxygenase-activating protein are localized in the nuclear envelope of activated human leukocytes. J. Exp. Med. 178:1935-1946.

4. Peters-Golden, M., and R.W. McNish. 1993. Redistribution of 5-lipoxygenase and cytosolic phospholipase $\mathrm{A}_{2}$ to the nuclear fraction upon macrophage activation. Biochem. Biophys. Res. Commun. 196:147-153.

5. Samuelsson, B. 1987. Leukotrienes and lipoxins: structure, biosynthesis, and biological effects. Science (Wash. DC). 237:1171-1175.

6. Bigby, T.D., and M.J. Holtzman. 1987. Enhanced 5-lipoxygenase activity in lung macrophages compared to monocytes from normal subjects. J. Immunol. 138:1546-1550.

7. Coffey, M.J., S.E. Wilcoxen, and M. Peters-Golden. 1994. Increases in 5-lipoxygenase activating protein expression account for enhanced capacity for 5-lipoxygenase metabolism that accompanies differentiation of peripheral blood monocytes into alveolar macrophages. Am. J. Respir. Cell Mol. Biol. 11: $153-158$.

7a. Ring, W.L., C.A. Riddick, J.R. Baker, D.A. Munafo, and T.D. Bigby. 1996. Human monocytes lose 5-lipoxygenase and its activating protein as they differentiate into monocyte-derived macrophages in vitro. Am. J. Physiol. (Cell Physiol.). In press.

8. Bennett, C.F., M.Y. Chiang, B.P. Monia, and S.T. Crooke. 1993. Regulation of 5-lipoxygenase and 5-lipoxygenase-activating protein expression in HL60 cells. Biochem. J. 289:33-39.

9. Steinhilber, D., O. Rådmark, and B. Samuelsson. 1993. Transforming growth factor $\beta$ upregulates 5-lipoxygenase activity during myeloid cell maturation. Proc. Natl. Acad. Sci. USA. 90:5984-5988.

10. Fair, A., and K.A. Pritchard. 1994. Oxidized low density lipoprotein increases U937 cell 5-lipoxygenase activity: induction of 5-lipoxygenase activating protein. Biochem. Biophys. Res. Commun. 201:1014-1020.

11. Meslier, N., A.J. Aldrich, and T.D. Bigby. 1992. Effect of interferon- $\gamma$ on the 5-lipoxygenase pathway of rat lung macrophages. Am. J. Respir. Cell Mol. Biol. 6:93-99.

12. Owen, W.F., M.E. Rothenberg, D.S. Silberstein, J.C. Gasson, R.L. Stevens, K.F. Austen, and R.J. Soberman. 1987. Regulation of human eosinophil viability, density, and function by granulocyte/macrophage colony-stimulating factor in the presence of 3T3 fibroblasts. J. Exp. Med. 166:129-141.

13. Rothenberg, M.E., W.F. Owen, D.S. Silberstein, J. Woods, R.J. Soberman, K.F. Austen, and R.L. Stevens. 1988. Human eosinophils have prolonged survival, enhanced functional properties, and become hypodense when exposed to human interleukin 3. J. Clin. Invest. 81:1986-1992.

14. Silberstein, D.S., W.F. Owen, J.C. Gasson, J.F. Dipersio, D.W. Golde, J.C. Bina, R. Soberman, K.F. Austen, and J.R. David. 1986. Enhancement of human eosinophil cytotoxicity and leukotriene synthesis by biosynthetic (recombinant) granulocyte-macrophage colony-stimulating factor. J. Immunol. 137:3290-3294.

15. Roubin, R., P.P. Elsas, W. Fiers, and A.J. Dessein. 1987. Recombinant tumor necrosis factor (rTNF) enhances leukotriene biosynthesis in neutrophils and eosinophils stimulated with the $\mathrm{Ca}^{2+}$ ionophore A23187. Clin. Exp. Immunol. 70:484-490.

16. Dahinden, C.A., J. Zingg, F.E. Maly, and A.L. de Weck. 1988. Leukotriene production in human neutrophils primed by recombinant human granulocyte-macrophage colony-stimulating factor and stimulated with the complement component C5a and FMLP as second signals. J. Exp. Med. 167:1281-1294.

17. Krump, E., and P. Borgeat. 1994. Kinetics of 5-lipoxygenase activation, arachidonic acid release, and leukotriene synthesis in human neutrophils: effects of granulocyte-macrophage colony-stimulating factor. Biochim. Biophys. Acta. 1213:135-139.

18. Palmantier, R., M.E. Surette, A. Sanchez, P. Braquet, and P. Borgeat 1994. Priming for the synthesis of 5-lipoxygenase products in human blood ex vivo by human granulocyte-macrophage colony-stimulating factor and tumor necrosis factor- $\alpha$. Lab. Invest. 70:696-704.

19. Pouliot, M., P.P. McDonald, L. Khamzina, P. Borgeat, and S.R. McColl. 1994. Granulocyte-macrophage colony-stimulating factor enhances 5-lipoxygenase levels in human polymorphonuclear leukocytes. J. Immunol. 152:851858

20. Pouliot, M., P.P. McDonald, P. Borgeat, and S.R. McColl. 1994. Granulocyte/macrophage colony-stimulating factor stimulates the expression of the 5lipoxygenase-activating protein (FLAP) in human neutrophils. J. Exp. Med. 179:1225-1232.

21. Stankova, J., M. Rola-Pleszczynski, and C.M. Dubois. 1995. Granulocyte-macrophage colony-stimulating factor increases 5-lipoxygenase gene transcription and protein expression in human neutrophils. Blood. 85:3719-3726.

22. Jakobsson, P.J., B. Odlander, and H.E. Claesson. 1991. Effects of monocyte-lymphocyte interaction on the synthesis of leukotriene $\mathrm{B}_{4}$. Eur. J. Biochem. 196:395-400.

23. Bøyum, A. 1968. Isolation of mononuclear cells and granulocytes from blood. Scand. J. Clin. Lab. Invest. 21(Suppl. 97):77.

24. Bradford, M.M. 1976. A rapid and sensitive method for the quantitation of microgram quantities of protein utilizing the principle of protein-dye binding. Anal. Biochem. 72:248-254.

25. Bigby, T.D., and N. Meslier. 1989. Transcellular lipoxygenase metabolism between monocytes and platelets. J. Immunol. 143:1948-1954.

26. Munafo, D.A., K. Shindo, J.R. Baker, and T.D. Bigby. 1994. Leukotriene $\mathrm{A}_{4}$ hydrolase in human bronchalveolar lavage fluid. J. Clin. Invest. 93: 1042-1050.

27. Sokal, R.R., and F.J. Rohlf. 1981. Biometry. W.H. Freeman, San Francisco, CA.

28. Hoshiko, S., O. Radmark, and B. Samuelsson. 1990. Characterization of the human 5-lipoxygenase gene promoter. Proc. Natl. Acad. Sci. USA. 87:90739077.

29. Kennedy, B.P., R.E. Diehl, Y. Boie, M. Adam, and R.A. Dixon. 1991. Gene characterization and promoter analysis of the human 5-lipoxygenase-activating protein (FLAP). J. Biol. Chem. 266:8511-8516.

30. Samuelsson, B., S. Hoshiko, and O. Radmark. 1991. Characterization of the promoter of the human 5-lipoxygenase gene. Adv. Prostaglandin Thromboxane Leukotriene Res. 21:1-8.

31. Schatz-Munding, M., and V. Ullrich. 1992. Priming of human polymorphonuclear leukocytes with granulocyte-macrophage colony-stimulation factor involves protein kinase $\mathrm{C}$ rather than enhanced calcium mobilisation. Eur. J. Biochem. 204:705-712. 
32. Surette, M.E., R. Palmantier, J. Gosselin, and P. Borgeat. 1993. Lipopolysaccharides prime whole human blood and isolated neutrophils for the increased synthesis of 5-lipoxygenase products by enhancing arachidonic acid availability: involvement of the CD14 antigen. J. Exp. Med. 178:1347-1355.

33. McDonald, P.P., M. Pouliot, P. Borgeat, and S.R. McColl. 1993. Induction by chemokines of lipid mediator synthesis in granulocyte-macrophage colony-stimulating factor-treated human neutrophils. J. Immunol. 151:6399-6409.

34. Piechele, G., S. Colli, E. Tremoli, and A. Maggi. 1993. 5-Lipoxygenase gene expression in HL60 cells during differentiation with DMSO. Pharmacol. Res. 27:53-60.

35. Elliott, M.J., M.A. Vadas, J.M. Eglinton, L.S. Park, L.B. To, L.G. Cleland, S.C. Clark, and A.F. Lopez. 1989. Recombinant human interleukin-3 and granulocyte-macrophage colony-stimulating factor show common biological effects and binding characteristics on human monocytes. Blood. 74:2349-2359.

36. Elliott, M.J., M.A. Vadas, L.G. Cleland, J.R. Gamble, and A.F. Lopez. 1990. IL-3 and granulocyte-macrophage colony-stimulation factor stimulate two distinct phases of adhesion in human monocytes. J. Immunol. 145:167-176.

37. Robin, G., S. Markovich, A. Athamna, and Y. Keisari. 1991. Human recombinant granulocyte-macrophage colony-stimulating factor augments viability and cytotoxic activities of human monocyte-derived macrophage in longterm cultures. Lymphokine Cytokine Res. 10:257-263.

38. Nissimov, R.D.L., and Y. Keisari. 1994. Effect of human recombinant granulocyte-macrophage colony-stimulating factor and IL-3 on the expression of surface markers of human monocyte-derived macrophages in long-term cultures. Lymphokine Cytokine Res. 13:239-245.

39. Miyajima, A., A.L.F. Mui, T. Ogorochi, and K. Sakamaki. 1993. Receptors for granulocyte-macrophage colony-stimulation factor, interleukin-3, and interleukin-5. Blood. 82:1960-1974.

40. Lopez, A.F., M.J. Elliott, J. Woodcock, and M.A. Vadas. 1992. GMCSF, IL-3 and IL-5: cross-competition on human haemopoietic cells. Immunol. Today. 13:495-500.

41. Lawrence, D.A. 1991. Identification and activation of latent transform- ing growth factor $\beta$. Methods Enzymol. 198:327-336.

42. Vitetta, E.S., R. Fernandez-Botran, C.D. Myers, and V.M. Sanders. 1989. Cellular interactions in the humoral immune response. Adv. Immunol. 45 $1-105$.

43. Shannon, M.F., S.R. Himes, and L.S. Coles. 1995. GM-CSF and IL-2 share common control mechanisms in response to costimulatory signals in $\mathrm{T}$ cells. J. Leukocyte Biol. 57:767-773.

44. Bonifati, C., M. Carducci, P. Cordiali Fie, E. Trento, G. Sacerdoti, M Fazio, and F. Ameglio. 1994. Correlated increases of tumour necrosis factor- $\alpha$, interleukin-6 and granulocyte monocyte-colony stimulating factor levels in suction blister fluids and sera of psoriatic patients-relationship with disease severity. Clin. Exp. Derm. 19:383-387.

45. Broide, D.H., M. Lotz, A.J. Cuomo, D.A. Coburn, E.C. Federman, and S.I. Wasserman. 1992. Cytokines in symptomatic asthma airways. J. Allergy Clin. Immunol. 89:958-967.

46. Marini, M., E. Avoni, J. Hollemborg, and S. Mattoli. 1992. Cytokine mRNA profile and cell activation in bronchoalveolar lavage fluid from nonatopic patients with symptomatic asthma. Chest. 102:661-669.

47. Kay, A.B., S. Ying, V. Verney, M. Gaga, S.R. Durhan, R. Moqbel, A.J. Wardlaw, and Q. Hamid. 1991. Messenger RNA expression of the cytokine gene cluster, interleukin 3 (IL-3), IL-4, IL-5, and granulocyte/macrophage colony-stimulating factor, in allergen-induced late-phase cutaneous reactions in atopic subjects. J. Exp. Med. 173:775-778.

48. Massey, W., B. Friedman, M. Kato, P. Cooper, A. Kagey-Sobotka, L.M. Lichtenstein, and R.P. Schleimer. 1993. Appearance of granulocyte-macrophage colony-stimulating factor activity at allergen-challenged cutaneous latephase reaction sites. J. Immunol. 150:1084-1092.

49. Walker, C., W. Bauer, R.K. Braun, G. Menz, P. Braun, F. Schwarz, T.T. Hansel, and B. Villiger. 1994. Activated T cells and cytokines in bronchoalveolar lavages from patients with various lung diseases associated with eosinophilia. Am. J. Respir. Crit. Care Med. 150:1038-1048. 